\title{
Computational intelligence for gas imports forecasting
}

\section{George Atsalakis*, Sofia Evangelia Ioannou and Constantin Zopounidis}

School of Production Engineering and Management, Technical University of Crete,

Crete, Greece

Email: gatsalakis@isc.tuc.gr

Email: sofia.ioannou91@gmail.com

Email: kostas@dpem.tuc.gr

*Corresponding author

\begin{abstract}
This paper investigates the ability to forecast the natural gas imports in Greece, using artificial intelligent methods. The Adaptive Neuro-Fuzzy Inference System (ANFIS) model and Artificial Neural Networks (ANN) model were developed. The overall gas import data required for the model were collected from Greek gas imports. The results of the developed models were compared with the actual data imports. Main statistical errors have been calculated in order to examine the forecasting accuracy of the proposed models. Further evaluation of the proposed models took place in comparison to the results with those of Autoregressive (AR) and Autoregressive Moving Average (ARMA). The results showed that gas import forecasting estimations using the ANFIS and ANN model were very encouraged. In terms of forecasting performance, it is clear from the empirical evidence that the ANFIS model outperforms artificial neural network and two other conventional models (AR and ARMA).
\end{abstract}

Keywords: neuro-fuzzy; ANFIS; artificial neural networks; fuzzy forecasting; ANFIS forecasting; neural network forecasting; import gas forecasting.

Reference to this paper should be made as follows: Atsalakis, G., Ioannou, S.E. and Zopounidis, C. (2015) 'Computational intelligence for gas imports forecasting', Int. J. Financial Engineering and Risk Management, Vol. 2, No. 1, pp.17-29.

Biographical notes: George Atsalakis is Lecturer of Technological Forecasting, at the School of Production Engineering and Management at Technical University of Crete, Greece. He received the Diploma in Business Administration from the Economical University of Athens, MSc degree in Business Administration and the $\mathrm{PhD}$ degree in Stock Market Forecasting, both from Technical University of Crete in 2000 and 2006, respectively. His research interests are focused in the areas of forecasting in the local and international markets, technological forecasting, forecasting of entrepreneurship evolution and forecasting of innovative economies and societies. He is using fuzzy, neuro-fuzzy and neural networks as modelling tools. He has published two books and over 90 book chapters, scientific journals and conference papers. 
Sofia Evangelia Ioannou is currently studying Engineering with Finance at the University College of London. She completed her Diploma in Production Engineering and Management from Technical University of Crete, Greece, in 2013. Her main courses are engineering management, project \& production management, engineering economics, financial management, marketing, thermodynamics, machine elements, simulation, quality control, and data analysis.

Constantin Zopounidis is Professor of Financial Engineering and Operations Research at the Technical University of Crete, Greece, Distinguished Research Professor at Audencia Nantes School of Management, France, Senior Academician of the Royal Academy of Economics and Financial Sciences of Spain, and member of the Royal Academy of Doctors of Spain. His research interests include financial engineering, financial risk management, and multiple criteria decision making. He has published over 300 papers in premier international journals, books, edited volumes, and conference proceedings. In recognition of his research work, he has received awards and honorary distinctions from several international research organisations. He is also the founder and elected president of the Financial Engineering and Banking Society.

This paper is a revised and expanded version of a paper entitled 'Comparison of neuro fuzzy logic (ANFIS) and artificial neural networks (ANN) in natural gas imports' presented at the '4th National Conference of the Financial Engineering and Banking Society', Athens, Greece, December 2013.

\section{Introduction}

Forecast modelling, like many other fields, has a great importance for the economic field and has been quite widely used. Many sectors make use of this forecasting modelling, ranging from forming decision-making mechanisms of economic units to forming and evaluating macroeconomic policies.

The import of natural gas in the energy balance of Greece has already affected major sectors of economic and social life of the country. It is expected that as even greater intrusion, as natural gas is the best source of primary energy, after renewable and the price ranges at lower levels than oil.

The aim of this research is to forecast the imports of natural gas in Greece using two flexible models (Adaptive Neuro-Fuzzy Inference System (ANFIS), Artificial Neural Networks (ANN)) and two conventional models. The comparison of the results of these four models reveals the superiority of the hybrid ANFIS model.

\subsection{Forecasting of energy imports and production}

Over the last few decades, there has been much research directed at forecasting the future and making better decisions. This research has led to many developments in forecasting methods. Most of these methodological advances have been based on statistical techniques. First of all, Martz et al. (1986) used Kalman filter in order to predict monthly 
US oil imports. They used the state equation ARIMA model and the observation equation using a regression of the revised, estimated and company-forecasted values on the true values. Marti used a method with the coefficient of concordance and evaluated waterborne New England's energy imports from 1994 to 2004 (Marti, 2008). In 2012 another analysis about the seasonality of China's crude oil import, in order to help the stakeholders with inventory control and production planning, used X-12-ARIMA method which was based in time series (Zhou and Dong, 2012). Lin and Wang forecasted the production and import of natural gas. Also, they used annual data and drawn conclusion using the logistic growth model and Gaussian curve model (Lin and Wang, 2012). Giant Gas Field (GGF) model was used by Soderberg et al. (2010) in order to analyse the Russian natural gas production and forecasted that major productions of Russian gas fields are in decline.

\subsection{Forecasting of energy consumption and demand}

The changes and developments in the world's economy have sharply increased energy consumption. It is important for proper energy planning of a country as well as the forecasting of consumption and demand. So, in 2001 there was an effort by Persaud and Kumar to apply the Oil and Gas Supply Model (OGSM) in Canada hence they gave projections of oil and natural gas supply and demand to the year 2020 (Persaud and Kumar, 2001). Many different models have been used in order to forecast the natural gas demand and consumption. In Bangladesh, the researchers forecasted the natural gas demand until 2023 using a dynamic econometric model which derives a relation between GPD and natural gas demand (Wadud et al., 2011). On the other hand, a mathematical model was used by Dalfard et al. (2013) to examine the relation between gas consumption and its prices. They used an integrated adaptive Fuzzy Inference System (FIS). Nevertheless, it suggested a novel procedure for construction and adaptation of Takagi-Sugeno Fuzzy Inference System (TS-FIS) in order to incorporate the impact of price hike into modelling. They also used ANFIS to forecast natural gas consumption in power plants.

Moreover, statistical method is an important way of predicting energy consumption. So, in 2008 there was a research about the estimation of individual residential and small commercial customers and the researchers used a statistical method based on non-linear regression principles (Vondracek et al., 2008). A different method referred to as the degree-day method was used for prediction of natural gas consumption for heating in Turkey in 2023 (Sarak and Satman, 2003). This method used some functions which calculated the fuel consumption for building heating purpose in a heating season and the total annual energy consumption of the city. It also examined the responsibility of the results comparing the real data with the forecasted. In China, people use natural gas more and more. So, it was important for government to forecast the yearly gas consumption. It used a model based on system dynamics model which was developed using STELLA software (Li et al., 2011). A model which was of the non-linear regression type, with individual customer-specific parameters that, nevertheless, had a common distribution corresponding to the non-linear mixed effects model framework was used which estimated the description and forecast of the daily consumption of natural gas at the level 
of individual customers (Brabec et al., 2008). Models like SARIMAX, ANN-MLP and ANN-RBF were used to forecast the daily natural gas consumption in Turkey utilising some meteorological data too (Taspinara et al., 2013). Both neural and abductive networks were used in a univariate modelling of monthly demand series based on data for earlier six years to forecast the energy demand of the seventh year (Abdel-Aal, 2008). Neural networks were also used to forecast gas consumption in Istanbul but this time with multivariate time series methods (Demirel et al., 2012). Forecasting of the gas consumption in Bahrain, Saudi Arabia, Syria and UAE for 2008-2015 were performed using a neuro-fuzzy-stochastic frontier analysis based in ANFIS. The results were compared with respect to MAPE (Azadeh et al., 2011). Wang et al. (1999) brought out the first metric model for forecasting natural gas consumption in China. After that, other researchers used more exotic models like Fourier functions (Jiao et al., 2002), grey neural network (Tan, 2003), combination models (Yin and Liu, 2004; Wan et al., 2007) or selforganising data mining method (Gao and Dong, 2008). All these models provided different ways to depict trends in natural gas consumption and were considered useful for planning gas industry development.

\section{Artificial neural networks}

ANN or simply Neural Networks (NN) are mathematical models for processing information that approximates the computational and representational ability through synapses. The model was inspired by bioelectrical networks generated in the brain between neurons (nerve cells) and synapses (contact points of nerve endings). Neurons are the building blocks of the network. There are two types of neurons, input neurons and computational neurons: Neurons input which do not calculate anything mediate between the inputs of the network and computational neurons. The computational neurons multiply the inputs with the condensed weights and calculate the sum of the product. Therefore, the result shows the argument of the transfer function.

The output $y_{k}$ of neuron $k$ is given using the equation:

$$
y_{k}=\varphi\left(\sum_{i=0}^{N} x_{k i} w_{k i}\right)
$$

where $x_{k i}$ is the $i$-th output of neuron $k, w_{k i}$ is the $i$-th weight of neuron $k$. The $w_{k 0}$ is called bias or threshold. The value of the input is always one $\left(x_{k 0}=1\right)$. If the total sum of the remaining inputs of neuron is greater than this value, the neuron is activated but if smaller, the neuron remains inactive.

The model of neural networks, as used even now was discovered by Widrow and Hoff (1962). They identified the perceptron as the adaptive element which is similar to the neuron. A neuron, the foundation of a neural information processing system, consists of:

- Cellbody containing a nucleon.

- Dendrites that supply the body with external signals.

- Axons that carry signals from the body to the other bodies. 
In other words, it can be translated into computer technology terms as follows:

- The piece of the core element, which is called perceptron, comprises of a summing element $\Sigma$ and a non-linear element NL.

- The multiple input signals $x_{i}$ are connected via weights, which can be adjusted and redefined, with the core of element.

- The output signal (can often be more than one) $y_{d}$.

Figure 1 Structure of neuron and percepton
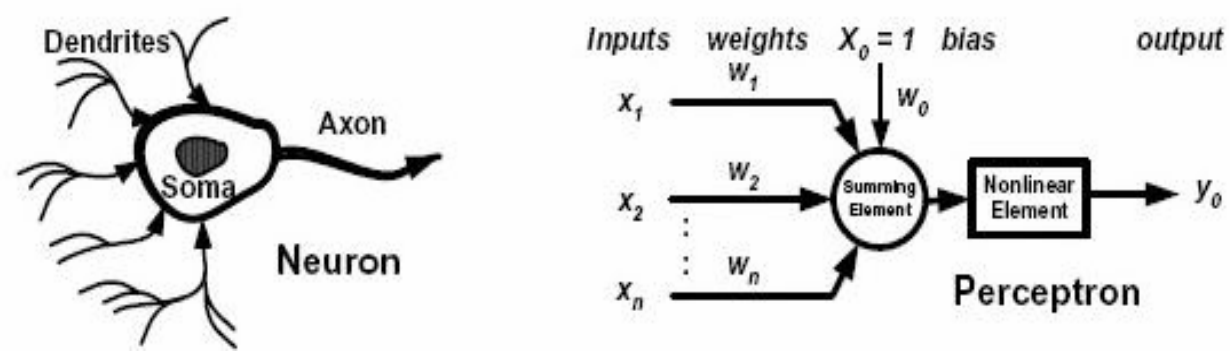

\section{Neuro-fuzzy systems}

Neuro-fuzzy refers to combinations of artificial neural networks and fuzzy logic. The structure of these systems is the fuzzy neurons. The form of fuzzy neurons was shown in Figure 2.

Figure 2 Fuzzy neuron

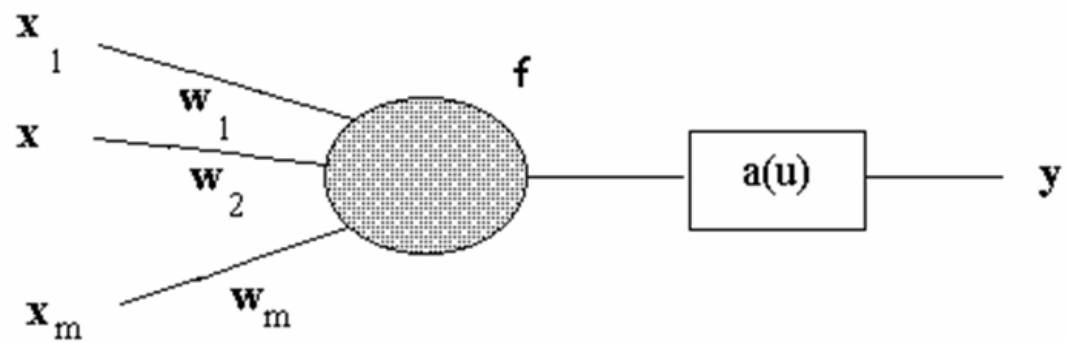

Notes: $\quad x_{1}, x_{2}, . ., x_{m}$ neuron inputs, $w_{1}, w_{2}, . ., w_{m}$ the weights, $f$ the membership function of neuron, $\alpha$ the activation function and $y$ neuron outputs.

\subsection{Adaptive neuro-fuzzy inference system (ANFIS)}

ANFIS is a neuro-fuzzy network which is proposed by Jang (1993), It was used in this study for the short-term forecasting of natural gas imports. The algorithm ANFIS is very 
important and is one of the first applied to solve problems in the field of neuro-fuzzy systems. ANFIS is based on Takagi-Sugeno fuzzy inference system. It integrates both neural networks and fuzzy logic principles and it has potential to capture the benefits of both in a single framework. Its inference system corresponds to a set of fuzzy IF-THEN rules that have learning capability to approximate non-linear functions. The following describes the network, assuming that the problem which is going to be solved has two inputs $(x, y)$ and one output $(z)$.

Assuming we have a first order Sugeno model, a standard rule base might be as follows:

Rule 1: If $x$ is $A_{1}$ and $y$ is $B_{1}$ then $f_{1}=p_{1} \times x+q_{1} \times y+r_{1}$

Rule 2: If $x$ is $A_{2}$ and $y$ is $B_{2}$ then $f_{2}=p_{2} \times x+q_{2} \times y+r_{2}$

The architecture of ANFIS is described using Figure 3.

Figure 3 Architecture of ANFIS

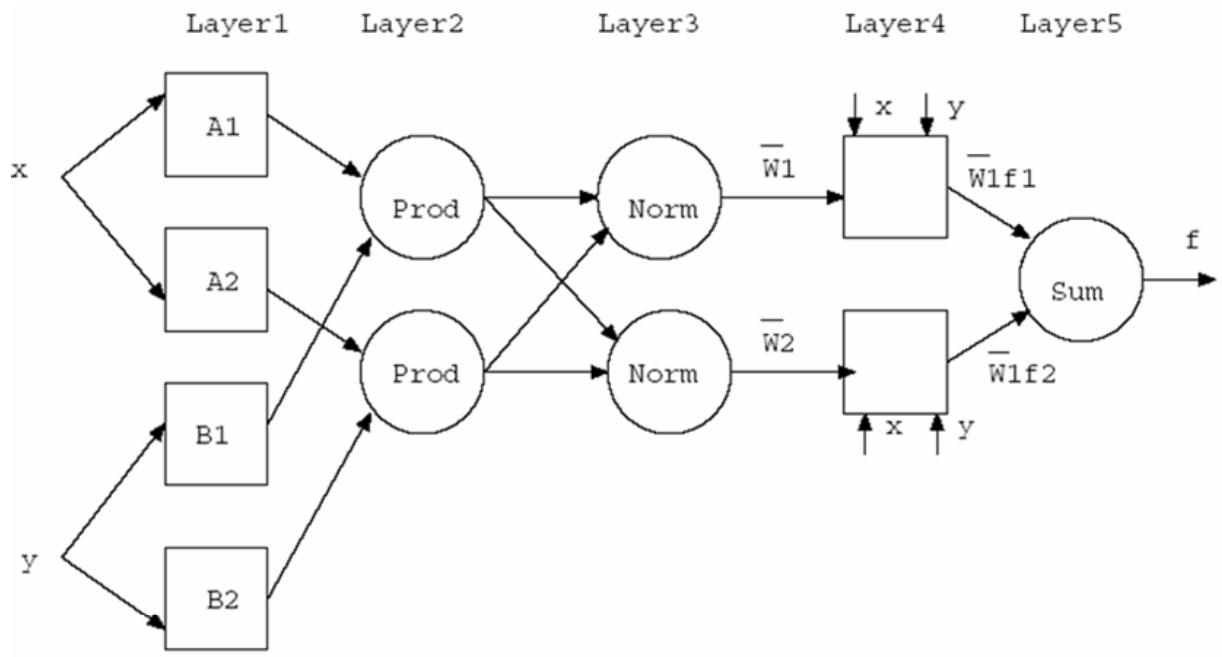

Each node and each level has a specific use. Every node $\mathrm{i}$ in first layer is an adaptive node with a node function $O_{i}^{1}(x)=\mu_{A_{A}}(x)$. Also, $\mathrm{x}$ is the input node $\mathrm{i}$ and $\mathrm{A}_{\mathrm{i}}$ is a linguistic label associated with this node. Therefore $O_{1, i}$ is the membership grade of a fuzzy set $\left(A_{1}, A_{2}, B_{1}, B_{2}\right)$. Usually, it selects $\mu_{A i}(x)$ which is bell-shaped with maximum equal to 1 and minimum to 0 , as the generalised bell function:

$$
\mu_{\mathrm{A}}(x)=\frac{1}{1+\left|\frac{x-c_{i}}{a_{i}}\right|^{2 b_{i}}}
$$

where $\alpha_{i}, b_{i}$ and $c_{i}$ are the parameter set. Parameters in layer 1 are referred to as premise parameters. 
In the second layer every node is a fixed node labelled Prod. Each node represents the fire strength of the rule. In addition, any other T-norm operator that perform the AND operator can be used. The output is the product of all the incoming signal:

$$
O_{i}^{2}=w_{i}=\mu_{A_{i}}(x) * \mu_{B_{i}}(y), i=1,2
$$

In layer 3 every node is a fixed node labelled Norm. The $i$-th node calculates the ratio of the $i$-th rule's firing strength to the sum of the firing strength of all rules. The output of each node in this layer is called normalised firing strengths and it is given by:

$$
\bar{w}_{i}=\frac{w_{i}}{w_{1}+w_{2}} i=1,2
$$

In the next layer (layer 4 ) every node is an adaptive node with a node function

$$
O_{i}^{4}=\bar{w}_{i}^{*} f_{i}=\bar{w}_{i}^{*}\left(p_{i}^{*} x+q_{i}^{*} y+r_{i}\right)
$$

where $\bar{w}_{i}$ is the normalised firing strength from layer 3 and $\left\{p_{i}, q_{i}, r_{i}\right\}$ is the parameter set of this node. These parameters are called consequent parameters.

In the last layer, layer 5 there is only one node which is a fixed node labelled sum and it computes the overall output as the summation of all incoming signals:

$$
\text { Overall output }=O_{i}^{5}=\sum_{i} \bar{w}_{i} * f_{i}=\frac{\sum_{i} w_{i}^{*} f_{i}}{\sum_{i} w_{i}}
$$

\section{Application and discussion}

To evaluate the performance of the models, an error analysis, using some well known statistical errors: i.e. Mean Square Error (MSE), Root Mean Square Error (RMSE), Mean Absolute Error (MAE) and Mean Absolute Percentage Error (MAPE) were carried out. The results of the ANFIS and ANN were further compared with those of an Autoregressive (AR) model and an Autoregressive Moving Average model (ARMA).

We extracted four types of errors with our program to make comparison between models AR, ARMA and ANFIS or ANN. The errors are:

1 Mean Square Error: $M S E=\frac{1}{N} \sum_{t=1}^{N} e_{t}^{2}$

2 Root Mean Square Error: $R M S E=\sqrt{\frac{\sum_{t=1}^{N} e_{t}^{2}}{N}}$

3 Mean Absolute Error: $M A E=\frac{1}{N} \sum_{t=1}^{N}\left|e_{t}\right|$

4 Mean Absolute Percentage Error: $M A P E=\frac{100}{N} \sum_{t=1}^{N}\left|\frac{F_{t}-A_{t}}{A_{t}}\right|$ 
The 1917 total observed daily data sets, starting from January 2008 to March 2013 were used throughout the training and evaluation phase. The 1532 (75\%) observations have been used to train the models. The remaining 385 (25\%) samples have been used to evaluate the models out of sample. Figure 4 depicts the raw data that have been used to train and evaluate the models.

Figure 4 An illustration of the row training data (see online version for colours)
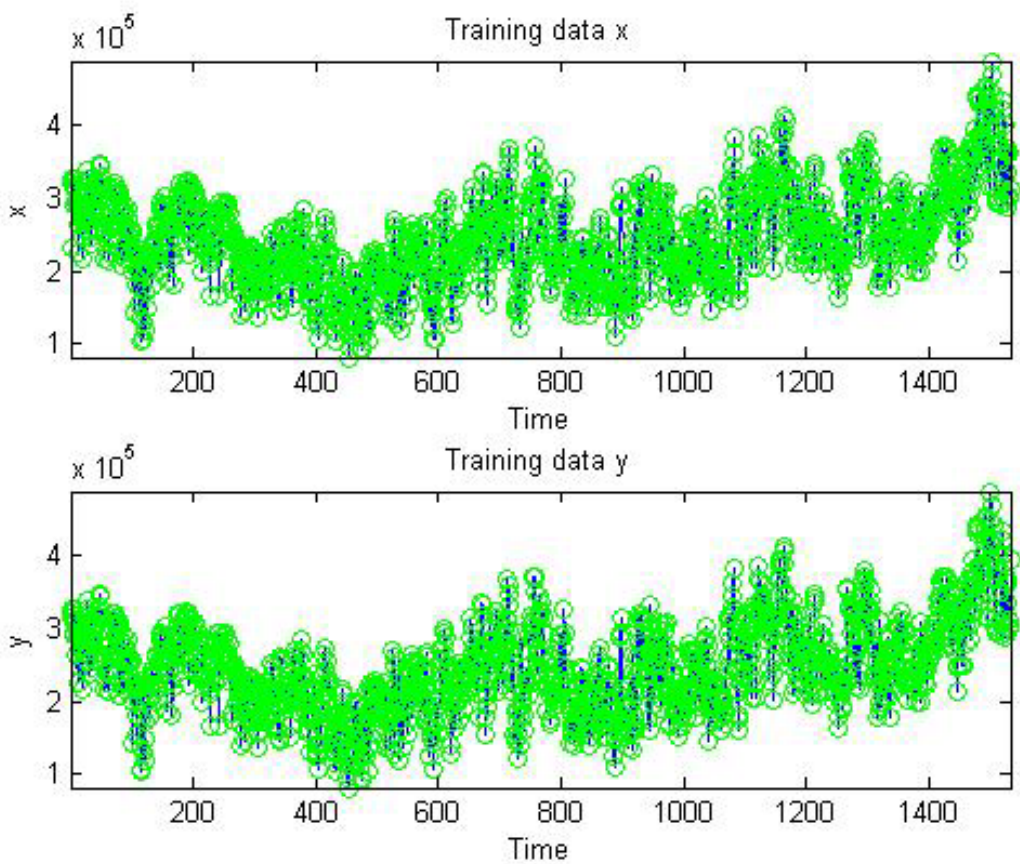

The same data were used to train and evaluate the ANFIS, the NN, the AR and ARMA forecasting model.

\subsection{Model presentation}

The initial parameters set in ANFIS model are $n=2$, step size 0.1 , epochs $=500$ and generalised bell membership function (gbellmf). After we ran the program with the above settings, we repeated the process for 100 and 250 epochs keeping gbellmf as the Membership Function (MF). Thereafter we ran our data again for 500, 100, 250 times, but we used all available membership functions which is gaussmf, Gaussian Membership Function (gauss2mf), Triangular Membership Function (trimf), Sigmoid Membership Function (smf), Trapezoidal Membership Function (trapmf), П-shaped Membership Function (pimf) and Z-shaped Membership Function (zmf).

The model was tested many times using different time epochs. Finally, the best results were obtained at 500 epochs using the generalised bell MF with small deviations in some results. Figure 5 depicts the form of the bell membership functions before and after training of the model. 
Figure 5 Generalised bell membership functions before and after training (see online version for colours)
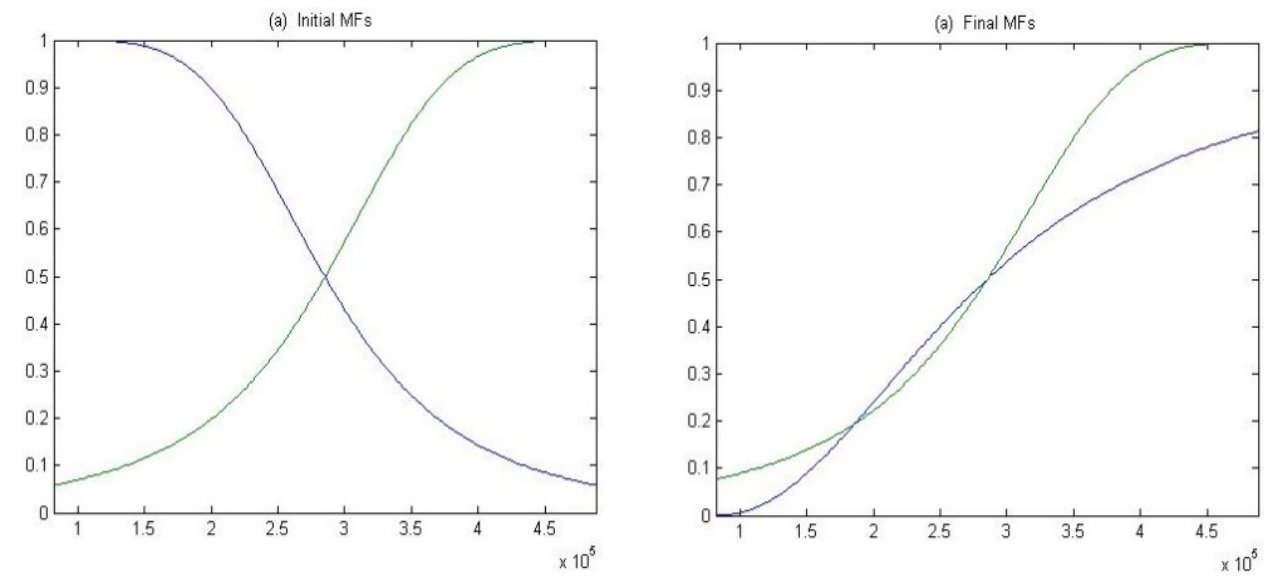

Figure 6 shows the RMSE and the step size against the number of training epochs, during the training phase. Figures 7-10 show a graphical representation of the forecasting results of the ANFIS, ANN, AR and ARMA models.

Figure 6 RMSE and step size during the training (see online version for colours)
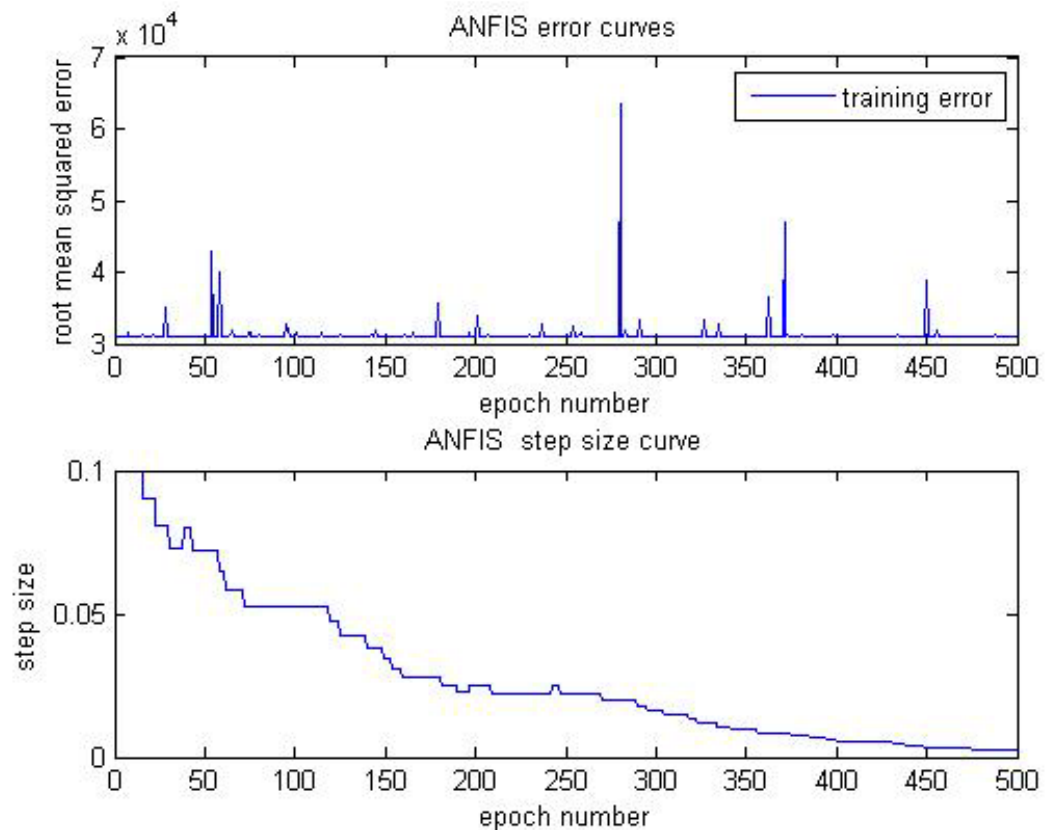
Figure 7 ANFIS out of sample forecasting results (see online version for colours)

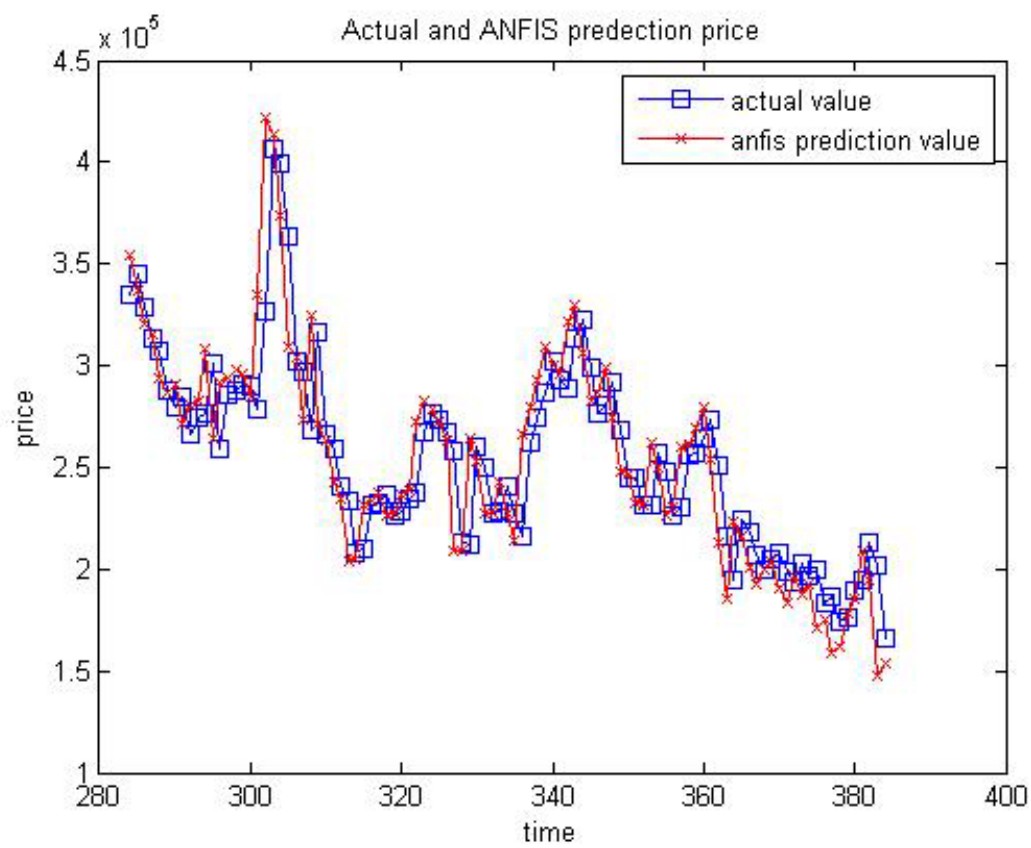

Figure 8 ANN forecasting values and actual values (see online version for colours)

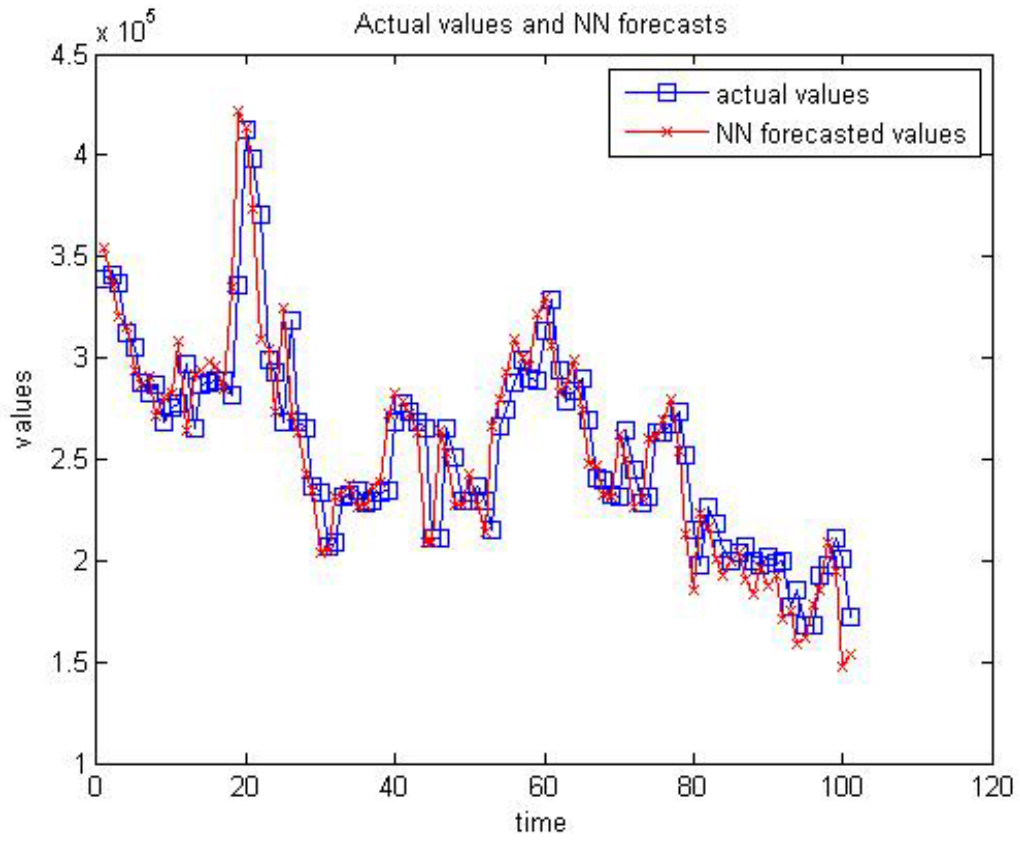


Figure 9 AR prediction values vs. actual values (see online version for colours)

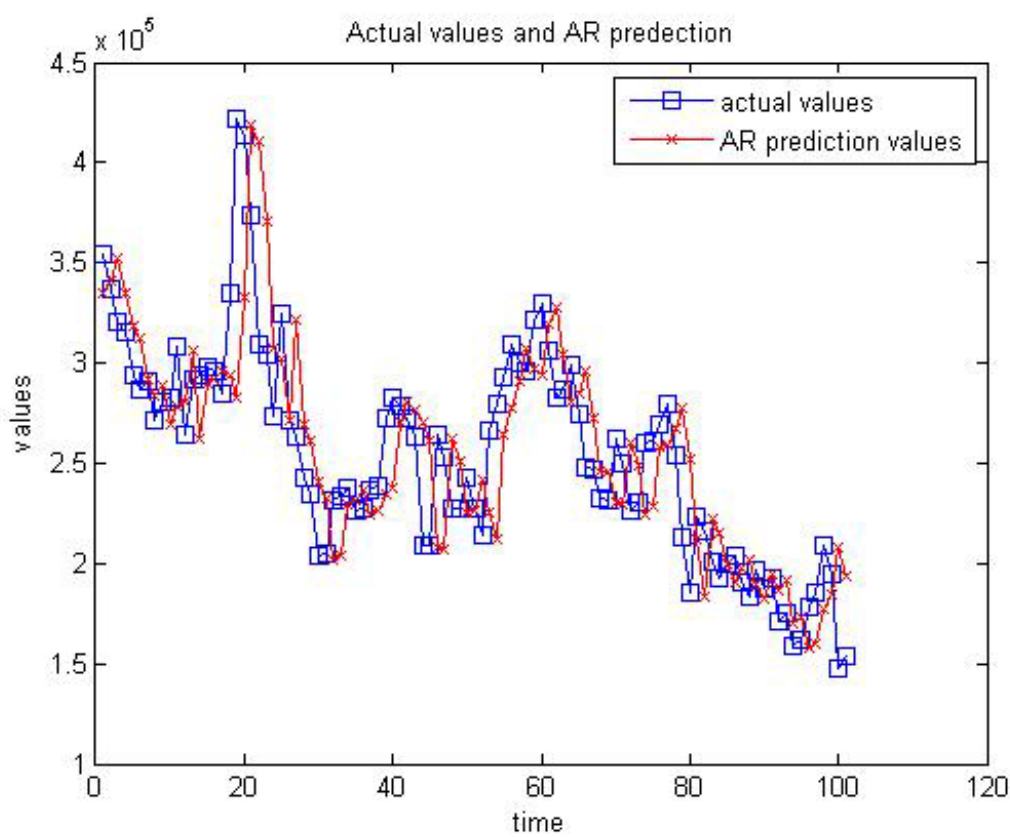

Figure 10 ARMA prediction values vs. actual values

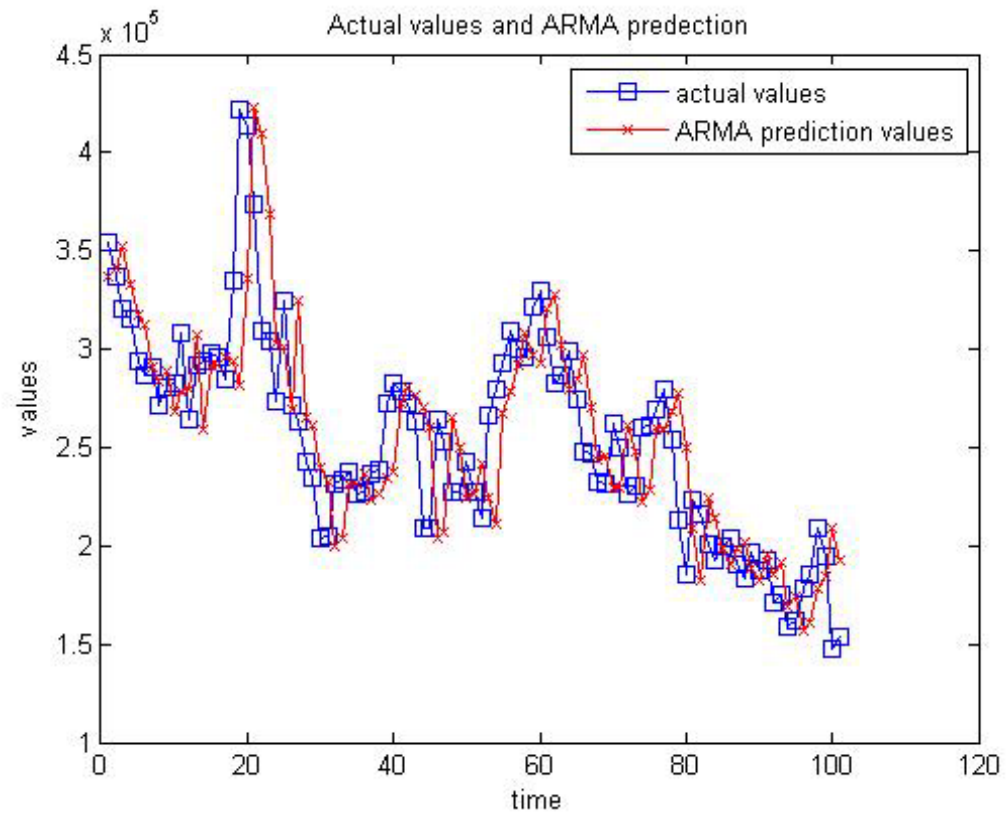


RMSE is the most important indicator which shows the effectiveness of forecasting model. So we compared the RMSE by selecting the lowest between ANFIS and ANN. The best results appeared in membership function gauss in conjunction with the forecasting model ANFIS. Table 1 illustrates the forecasting results of four models. It is worthy to mention that the models were trained using non-transformed data. Thus, the models have shown significant variations in the errors of the performance evaluation for the four models.

Table 1 Errors for one day ahead forecasting results

\begin{tabular}{ccccc}
\hline & $A N F I S$ & $N N$ & $A R$ & $A R M A$ \\
\hline MSE & 0.572681007411595 & 0.580812764784016 & 1.707648138477331 & 1.331831474524326 \\
RMSE & 0.000023930754426 & 0.000024100057361 & 0.000041323699477 & 0.000036494266324 \\
MAE & 0.000018525059142 & 0.000018721290945 & 0.000029495080098 & 0.000028534765456 \\
MAPE & 0.000000008345479 & 0.000000008405670 & 0.000000012798825 & 0.000000012541864 \\
\hline
\end{tabular}

Hence, it appears that the ANFIS model were more accurate, where all the values of errors were smaller. It also indicates that the lower value of the RMSE was 0.000023930754426 in the ANFIS model and the highest value of the RMSE was 0.000041323699477 in the AR model. While, the values of the MAPE 0.000000008345479 in the ANFIS forecasting model are much lower than those derived in the other models. In addition, the values of the MSE 0.572681007411595 and MAE 0.000018525059142 in the ANFIS model are lower than those found in the result of the other models.

\section{Conclusion}

This study was based on the comparative analysis of neuro-fuzzy network, an artificial neural network and two conventional forecasting methods. Four different models were investigated, namely, ANFIS, NN, AR and ARMA. Thus, this research aimed at proving that a neuro-fuzzy approach can be used to forecast the daily natural gas imports. In addition, the weak aspects of other forecasting methodologies for time series could be overcome with the proposed ANFIS.

Through this research, we showed that the introduction of natural gas on a daily basis can be captured by an ANFIS system which can help to forecast the short-term gas import quantities. The results are very encouraging and have shown that the ANFIS model performs better than the other conventional methods of forecasting and its rules are simpler and training time was smaller. Therefore, it means that the ANFIS model can successfully be employed in gas strategic planning by a decision maker.

\section{References}

Abdel-Aal, R.E. (2008) 'Univariate modeling and forecasting of monthly energy demand time series using abductive and neural networks'. Computers \& Industrial Engineering, Vol. 54, No. 4, pp.903-917.

Azadeh, A.A., Asadzadeh, S.M., Saberi, M.M., Nadimi, V.V., Tajvidi, A.A. and Sheikalishahi, M.M. (2011) 'A Neuro-fuzzy-stochastic frontier analysis approach for long-term natural gas consumption forecasting and behavior analysis: the cases of Bahrain, Saudi Arabia, Syria, and UAE', Applied Energy, Vol. 88, No. 11, pp.3850-3859. 
Brabec, M., Konár, O., Pelikán, E. and Malý, M. (2008) 'A nonlinear mixed effects model for the prediction of natural gas consumption by individual customers', International Journal of Forecasting, Vol. 24, No. 4, pp.659-678.

Dalfard, V.M., Asli, M.N., Asadzadeh, S.M., Sajjadi, S.M. and Nazari-Shirkouhi, A. (2013) 'A mathematical modeling for incorporating energy price hikes into total natural gas consumption forecasting', Applied Mathematical Modelling, Vol. 37, No. 8, pp.5664-5679.

Demirel, Ö., Zaim, S., Çalişkan, A. and Özuyar, P. (2012) 'Forecasting natural gas consumption in Istanbul using neural networks and multivariate time series methods', Turkish Journal of Electrical Engineering \& Computer Sciences, Vol. 20, No. 5, pp.695-711.

Gao, J. and Dong, X.C. (2008) 'Natural gas forecast with self-organizing data mining', Statistics and Decision, Vol. 7, pp.50-52.

Jang, J.S. (1993) 'ANFIS: adaptive-network-based fuzzy inference systems', IEEE Transactions on Systems Man \& Cybernetics, Vol. 23, No. 3, pp.665-685.

Jiao, W.L., Jin, J.B., Lian, L.M. and Cui, J.H. (2002) 'Time series model of predicting short period city gas load', Natural Gas Industry, Vol. 22, pp.92-94.

Li, J., Dong, X., Shangguan, J. and Höök, M. (2011) 'Forecasting the growth of Chinese natural gas consumption', Energy, Vol. 36, No. 3, pp.1380-1385.

Lin, B. and Wang, T. (2012) 'Forecasting natural gas supply in China: production peak and import trends', Energy Policy, Vol. 49, pp.225-233.

Marti, B.E. (2008) 'Assessing new England's waterborne energy imports (1995-2004)', Marine Policy, Vol. 32, No. 4, pp.740-748.

Martz, H., Burris, A-H., Bruckner, L. and Bivins, R. (1986) 'Kalman filter estimation of monthly U.S oil imports', Energy, Vol.11, No. 3, pp.271-280.

Persaud, A.J. and Kumar, U. (2001) 'An eclectic approach in energy forecasting: a case of Natural Resources Canada's (NRCan's) oil and gas outlook', Energy Policy, Vol. 29, No. 4, pp.303-313.

Sarak, H. and Satman, A. (2003) 'The degree-day method to estimate the residential heating natural gas consumption in Turkey: a case study', Energy, Vol. 28, No. 9, pp.929-939.

Soderbergh, B., Jakobsson, K. and Aleklett, K. (2010) 'European energy security: analysis is of future Russian natural gas production and exports', Energy Policy, Vol. 38, No. 12, pp.7827-7843.

Tan, Y.F. (2003) 'Establishment and solution about forecast model of city gas seasonal load', Gas and Heat, Vol. 3, pp.131-133.

Taspinara, F., Celebi, N. and Tutkun, N. (2013) 'Forecasting of daily natural gas consumption on regional basis in Turkey using various computational methods', Energy and Buildings, Vol. 56, pp.23-31.

Vondracek, J., Cermakova, J., Eben, K., Brabec, M., Konár, O., Pelikán, E. and Malý, M. (2008) 'A statistical model for the estimation of natural gas consumption', Applied Energy, Vol. 85, No. 5, pp.362-370.

Wadud, Z., Dey, H.S., Kabir, M.A. and Khan, S. (2011) 'Modeling and forecasting natural gas demand in Bangladesh', Energy Policy, Vol. 39, No. 11, pp.7372-7380.

Wan, X., Li, B.W., Yan, Y. and Jiang, J.D. (2007) 'Study on combined forecasts of Chongqing's natural gas market demand during 11th five-year plan period', Natural Gas Technology, Vol. 1, pp.82-84.

Wang, Y.C., Li, J.M., Zhu, Y. and Ren, Q.R. (1999) 'A new method to predict the dynamic demand of gas consumption', Petroleum Planning and Engineering, Vol. 9, pp.87-89.

Widrow, B. and Hoff, M.E. (1962) 'Associative storage and retrieval of digital information in networks of adaptive "neurons", Biological Prototypes and Synthetic Systems, Vol. 1, No. 160

Yin, J.C. and Liu, Z.B. (2004) 'Study on prediction model of gas demand self-adapting optimization combination', Natural Gas Industry, Vol. 24, pp.167-169.

Zhou, Z-B. and Dong, X-C. (2012) 'Analysis about the seasonality of China's crude oil import based on X-12-ARIMA', Energy, Vol. 42, No. 1, pp.281-288. 